\title{
EVALUACIÓN DE Cryptosporidium parvum COMO FACTOR DE RIESGO PARA LA PRESENTACIÓN DE DIARREA NEONATAL EN ALPACAS EN CUSCO
}

\author{
CRYPtosporidium parVum as Risk FaCtor on Neonatal Diarrhea in Alpacas of \\ Cusco
}

\section{Cecilia Villacorta G. ${ }^{1}$, Teresa López U. ${ }^{1,2}$, Armando González Z.$^{3}$ y Luis Gómez.$^{3}$}

\section{Resumen}

\begin{abstract}
El presente estudio tuvo como objetivo evaluar si la presencia de Cryptosporidium parvum es un factor de riesgo de diarrea en alpacas neonatas menores de 15 días de edad, provenientes de diversas unidades alpaqueras del departamento de Cusco. Se empleó el diseño epidemiológico de Caso-Control para establecer si existe una relación causal. Se tomaron 248 y 231 muestras fecales de animales con y sin diarrea, respectivamente, durante la temporada de parición del 2006. Se determinó la presencia de C. parvum mediante le técnica de tinción de Ziehl Neelsen Modificado (ZNM). Los datos se analizaron empleando una regresión logística, que ajustó variables potencialmente confundentes como edad, sexo, raza y lugar de origen de las alpacas. Se encontró un Odds Ratio de 4.3 (I.C. $=2.3-7.9)$. El estudio demuestra que las alpacas positivas a $C$. parvum tienen 4.3 veces mayor predisposición a sufrir diarreas en relación a las alpacas con diagnóstico ZNM negativo. Así mismo, se determinó asociación estadística significativa entre animales con infección por el parásito y los que manifiestan cuadros de diarrea $(23.4 \%$; $=58)$ en comparación con el grupo aparentemente sano $(8.6 \%$; $n=20)$.
\end{abstract}

Palabras clave: Cryptosporidium parvum, factor de riesgo, alpacas

\section{AbSTRACT}

The objective of the present study was to evaluate the presence of Cryptosporidium parvum as a risk factor to the occurrence of diarrhea in alpacas younger than 15 days of age from peasant communities in the area of Cusco, using a case-control study design. Stool samples were collected from animals with $(n=248)$ and without diarrhea $(n=231)$ during the 2006 calving season. The presence of $C$. parvum was determined by the Modified Ziehl Neelsen stain technique (MZN). The results were analyzed using a logistic regression model, adjusting potentially confusing variables as age, sex, breed and location of alpacas. The Odds Ratio was 4.3 (C.I. $=2.3-7.9)$. The study demonstrated that neonatal alpaca positives to C. parvum have 4.3 times higher predisposition to suffer diarrheas in

\footnotetext{
1 Laboratorio de Microbiología y Parasitología Veterinaria, ${ }^{3}$ Laboratorio de Medicina Veterinaria Preventiva, Facultad de Medicina Veterinaria, Universidad Nacional Mayor de San Marcos, Lima

2 E-mail:mtlu@terra.com.pe
} 
relation to the animals negative to MZN. In addition, significant association was observed between the animals MZN positive and the presence of diarrhea $(23.4 \%$; $\mathrm{n}=58)$ in comparison with the healthy group $(8.6 \% ; \mathrm{n}=20)$.

Key words: Cryptosporidium parvum, risk factor, alpacas

\section{INTRODUCCIÓN}

La crianza de alpacas constituye una importante fuente de ingresos, tanto para las empresas asociativas como para las pequeñas comunidades de la región sur del Perú. $\mathrm{Su}$ importancia radica en la producción de fibra y carne (Bustinza, 2001). Adicionalmente, la elaboración de cueros y pieles de alpaca para actividades artesanales constituyen una fuente de trabajo importante. Por otro lado, las alpacas son susceptibles a un gran número de enfermedades cuya importancia varía de acuerdo al sistema de crianza y al fundo alpaquero. En este sentido, los beneficios de la crianza de la alpaca se ven mermados por una serie de problemas sanitarios, en especial los que afectan a los recién nacidos (FAO, 2005).

Los problemas entéricos tienen un origen que frecuentemente es de carácter multifactorial. En este sentido, microorganismos como Clostridium perfringes tipo A y C, Escherichia coli, rotavirus, coronavirus, Cryptosporidium sp. y otros, pueden estar involucrados como único agente o en asociación (Holland, 1990). Uno de los principales enteropatógenos asociados a la alta morbilidad y baja mortalidad en alpacas neonatas es Cryptosporidium parvum (Rojas, 2004). En las alpacas, al igual que en otros rumiantes, la criptosporidiosis cursa con depresión, anorexia, deshidratación, dolor abdominal fiebre y, notoriamente, con diarrea (López et al., 2001).

Existen diversos estudios de prevalencia de la criptosporidiosis realizados en alpacas de 1 a 15 días de edad en varias lo- calidades del Perú, con prevalencias de 10 a $26 \%$. Asimismo, se han realizado estudios ecológicos de tipo transversal que establecieron una asociación temporal entre diarrea y presencia de Cryptosporidium (López, 1997; Romero, 1998).

Los estudios de prevalencia y los ecológicos de tipo transversal son un primer paso en el estudio de cualquier enfermedad, aunque tienen ciertas limitaciones. Los estudios ecológicos de tipo transversal calculan una asociación temporal, que no es suficiente para establecer una relación causal. La causalidad requiere, entre otras cosas, temporalidad, vale decir, que la causa ocurra primero. La temporalidad en condiciones de campo es difícil de establecer, de ahí que se empleen diseños específicos, tales como cohortes y caso-control. El presente estudio tuvo como objetivo evaluar la presencia de C. parvum como factor de riesgo para la presentación de diarrea neonatal en alpacas menores de 15 días de edad en la provincia de Canchis, departamento de Cusco, mediante un estudio epidemiológico de caso-control.

\section{Materiales y Métodos}

\section{Lugar de Estudio y Animales}

El muestreo se realizó en las localidades de Chillihua y La Raya, que corresponden al distrito de Maranganí, provincia de Canchis, Cusco, durante la temporada de parición, entre los meses de febrero y marzo de 2006. En Chillihua se muestreó los sectores de Chiaraje, Piti, Kero Viluyo, Pampalacaya y Velacunca; y en La Raya se muestreó el sector de La Raya-IVITA (Ins- 
tituto Veterinario de Investigaciones Tropicales y de Altura) y la Raya-UNSAAC (Universidad Nacional de San Antonio de Abad del Cusco).

El cálculo del tamaño muestral se hizo en base a la fórmula para estudios de casocontrol no pareado (Rothman y Greenland, 1998). Se utilizó un nivel de confianza de $95 \%$ y un poder de la prueba de $90 \%$. La proporción esperada en el grupo control expuesto fue de $11.36 \%$ (Foroca et al., 2001) y el Odds Ratio usado en el cálculo fue de 2.38 (López, 1997). Se determinó que el número mínimo de muestras requeridas para obtener un resultado confiable fuera de 163 , tanto para diarrea positivo como diarrea negativo; sin embargo, debido a la disponibilidad de animales, se colectaron 248 muestras fecales de animales con diarrea y 231 muestras fecales de animales sin diarrea, elegidos al azar. Se consideró que una alpaca estaba con diarrea cuando había presencia de heces líquidas o pastosas en la zona perineal y signos de deshidratación o depresión.

La edad de las crías de las alpacas no excedió los 15 días de edad (la mayoría estuvo entre los 13 y 15). El Cuadro 1 muestra el número de muestras fecales obtenidas en cada localidad y sector. Así mismo, en el momento del muestreo se registró datos de interés epidemiológico como edad, sexo, raza y área de procedencia de los animales.

\section{Manejo de las Muestras}

Las muestras fecales se recolectaron directamente del recto en bolsas plásticas rotuladas. Se hizo una extensión de cada muestra sobre 2 láminas portaobjetos, que se fijaron con metanol absoluto durante $5 \mathrm{~min}$ en un vaso kopling y luego se dejaron secar al ambiente. Las láminas fueron trasladadas al Laboratorio de Parasitología de la Facultad de Medicina Veterinaria de la Universidad Nacional Mayor de San Marcos, en Lima.
Las muestras se tiñeron empleando la coloración de Ziehl Neelsen Modificado (ZNM) (Henricksen y Pohlenz, 1981), y se observaron al microscopio con objetivo de 40X. Las láminas positivas se evaluaron a $400 \mathrm{X}$ y a $1000 \mathrm{X}$ para confirmar su diagnóstico. Se consideró el número de ooquistes por campo y la medida (largo por ancho).

Las muestras fueron consideradas positivas ante la presencia de ooquistes de $C$. parvum. Estos se visualizaron como organismos esféricos u ovalados de 4 a 6 micras de diámetro (Fayer et al., 2000; Xiao et al., 2004), color rojo fucsia con algunas granulaciones oscuras en su interior, que contrastaban con un fondo teñido de verde (Henricksen y Pohlenz, 1981).

\section{Análisis de Datos}

La evaluación de C. parvum como factor de riesgo para la presentación de diarrea neonatal en alpacas se midió mediante el cálculo de estimación del riesgo (Odds Ratio). Para ello se empleó el modelo de regresión logística múltiple para caso-control, utilizando el paquete estadístico STATA v. 8.0. Se calculó el riesgo de diarrea asociada a Criptosporidiosis ajustado a variables potencialmente confundentes como edad, sexo, raza y el lugar de origen de las alpacas muestreadas. Todos los resultados fueron interpretados con un intervalo de confianza de $95 \%$.

\section{Resultados y Discusión}

Se encontró una frecuencia de $16.3 \pm$ $3 \%$ positivas a $C$. parvum en las 479 muestras analizadas. Del total de animales positivos, el $74.4 \pm 9 \%$ presentaba diarrea lo cual evidencia que la criptosporidiosis tiene mayor predisposición a presentar casos diarreicos en alpacas neonatas. Por otro lado, el $47.4 \pm 4 \%$ de animales negativos a $C$. parvum presentó un cuadro diarreico en el 
Cuadro 1. Distribución del número de muestras fecales de alpacas neonatas recolectadas en las localidades de Chillihua y La Raya del distrito de Maranganí, provincia de Canchis, Cusco (2006)

\begin{tabular}{clc}
\hline Localidad & Sector & Número de muestras \\
\hline Chillihua & Piti & 9 \\
& Chiaraje & 26 \\
& Kero Viluyo & 30 \\
& Velacunca & 34 \\
& Pampalacaya & 122 \\
& IVITA & 62 \\
La Raya & UNSAAC & 196 \\
\hline Total & & 479 \\
\hline
\end{tabular}

momento de la toma de la muestra (Cuadro 2). Esto podría deberse a otros agentes etiológicos como bacterias y virus que cursan con este signo (Ortega-Mora et al., 1993). Hallazgos similares fueron encontrados en brotes diarreicos en ovinos recién nacidos y en crías de alpacas (López, 1997).

El sector de Chiaraje fue el que presentó mayor porcentaje de animales diarreicos por localidad, seguido por Piti y La RayaUNSAAC. Así mismo, no hubo diferencias entre machos y hembras, y las alpacas Suri mostraron una mayor frecuencia de heces diarreicas (58.9\%) que las Huacaya (40.5\%) (Cuadro 2).

Se aprecia que del total de muestras fecales aparentemente normales, el $8.6 \pm 4 \%$ (20/231) resultaron positivos a la infección (Cuadro 3). Estos animales se estarían comportando como portadores asintomáticos de la enfermedad (Lorenzo-Lorenzo et al., 1993). Este es un factor importante desde el punto de vista epidemiológico, sobre todo durante la parición, cuando el porcentaje de animales receptivos es muy elevado, representando un peligro potencial para las crías recién nacidas (Ortega-Mora, 1996; López, 1997).
El Odds Ratio o riesgo para evaluar el comportamiento de determinadas variables predictoras como presencia de C. parvum, edad, sexo, raza y lugar de origen para la presentación de diarrea neonatal en alpaca, ajustado a las variables potencialmente confundentes se muestra en el Cuadro 4.

Las alpacas con muestras ZNM positivo al parasito tienen 4.3 veces más probabilidades de presentar diarrea en relación a las alpacas con muestras ZNM negativo, ajustado al resto de variables predictoras, según lo indica los resultados del Odds Ratio (Cuadro 3). Ello evidencia que el C. parvum representa un factor de riesgo para la presentación de diarrea en crías de alpacas menores de 15 días en el departamento de Cusco. Este riesgo es considerado relativamente alto en comparación al OR de 2.38 encontrado por López (1997) en un estudio transversal de tipo ecológico. Este resultado, además, sugiere un riesgo potencial de infección para las personas que laboran con estos animales, debido a que este parásito ha sido reconocido como una entidad zoonótica (Acha y Szyfres, 2003). 
Cuadro 2. Distribución de alpacas neonatales de las localidades de Chillihua y La Raya del distrito de Maranganí, provincia de Canchis, Cusco con presencia de diarrea, según la presencia de Cryptosporidium parvum, procedencia, sexo y raza (2006)

\begin{tabular}{|c|c|c|c|c|}
\hline \multirow{2}{*}{ Variable } & & \multirow{2}{*}{$\begin{array}{l}\text { Animales } \\
\text { muestreados } \\
\text { (n) }\end{array}$} & \multicolumn{2}{|c|}{ Presencia de diarrea } \\
\hline & & & $\mathrm{N}^{\mathrm{o}}$ & $\%$ \\
\hline \multicolumn{5}{|c|}{ C. parvum } \\
\hline & Positivo & 78 & 58 & $74.4 \pm 9$ \\
\hline & Negativo & 48 & 190 & $47.4 \pm 4$ \\
\hline \multicolumn{5}{|c|}{ Procedencia } \\
\hline & Chiaraje & 26 & 19 & $73.1 \pm 2$ \\
\hline & Piti & 9 & 6 & $66.6 \pm 3$ \\
\hline & UNSAAC & 196 & 114 & $58.1 \pm 7$ \\
\hline & Pampalacaya & 122 & 64 & $52.4 \pm 9$ \\
\hline & Kero Viluyo & 30 & 14 & $46.6 \pm 2$ \\
\hline & IVITA & 62 & 23 & $37.1 \pm 2$ \\
\hline & Velacunca & 34 & 8 & $23.5 \pm 2$ \\
\hline \multicolumn{5}{|l|}{ Sexo } \\
\hline & Macho & 254 & 128 & $50.4 \pm 6$ \\
\hline & Hembra & 225 & 120 & $53.3 \pm 6$ \\
\hline \multicolumn{5}{|l|}{ Raza } \\
\hline & Suri & 112 & 66 & $58.9 \pm 9$ \\
\hline & Huacaya & 367 & 182 & $49.5 \pm 5$ \\
\hline Total & & 479 & 248 & $51.7 \pm 4$ \\
\hline
\end{tabular}

Cuadro 3. Distribución de alpacas neonatales de las localidades de Chillihua y La Raya del distrito de Maranganí, provincia de Canchis, Cusco con presencia de diarrea, según la presencia de Cryptosporidium parvum (2006)

\begin{tabular}{|c|c|c|c|}
\hline \multirow[t]{2}{*}{ Tipo de heces } & \multirow{2}{*}{$\begin{array}{l}\text { Animales } \\
\text { muestreados } \\
\text { (n) }\end{array}$} & \multicolumn{2}{|c|}{ Positivo a Cryptosporidium parvum } \\
\hline & & $\mathrm{N}^{\mathrm{o}}$ & $\%$ \\
\hline Sanas & 231 & 20 & $8.6 \pm 4$ \\
\hline Diarreicas & 248 & 58 & $23.4 \pm 5$ \\
\hline Total & 479 & 78 & $16.3 \pm 3$ \\
\hline
\end{tabular}


Cuadro 4. Regresión logística múltiple (MLG) interacción entre las diferentes variables estudiadas versus la presencia de diarrea neonatal en alpacas provenientes del departamento de Cusco (2006)

\begin{tabular}{|c|c|c|c|}
\hline Diarrea & OR & $p$ & (I.C. $95 \%$ ) \\
\hline Diag. positivo a $C$. parvum & 4.3 & $<0.001$ & $2.3-7.9$ \\
\hline $\mathrm{Raza}^{1}$ & 0.6 & 0.05 & $0.4-1.0$ \\
\hline $\mathrm{Sexo}^{2}$ & 1.1 & 0.39 & $0.8-1.7$ \\
\hline Edad (días) & 1.0 & 0.37 & $0.9-1.1$ \\
\hline \multicolumn{4}{|l|}{ Rebaño ${ }^{3}$} \\
\hline Pitti & 5.1 & 0.04 & $1.1-23.5$ \\
\hline Chiaraje & 4.4 & 0.008 & $1.5-13.2$ \\
\hline La Raya - UNSAAC & 2.9 & 0.001 & $1.5-5.6$ \\
\hline Pampalacaya & 2.0 & 0.04 & $1.1-4.1$ \\
\hline Kero Viluyo & 1.9 & 0.17 & $0.8-4.9$ \\
\hline Velacunca & 0.43 & 0.11 & $0.15-1.2$ \\
\hline
\end{tabular}

${ }^{1}$ Suri y Huacaya. Se tomó como referencia a la Suri

${ }^{2}$ Hembra y macho. Se tomó de referencia el macho

${ }^{3}$ Se tomó de referencia el rebaño de La Raya - IVITA

La variable sexo no presentó asociación estadística, lo que evidencia que la diarrea neonatal no tiene predilección por sexo alguno. Hallazgos similares fueron reportados en crías de alpacas (Caman, 1996; Tribeño, 1997), en bovinos y caprinos (Rajkhowa et al., 2006) y en niños (Kuhls et al., 1994). Por otro lado, la variable edad no presentó asociación significativa, posiblemente debido a que no hubo una distribución uniforme de la edad, al provenir la mayor parte de las muestras de animales de 13 a 15 días.

Los sectores Piti y Chiaraje de la localidad de Chillihua presentaron el mayor riesgo para la presentación de diarrea, ajustado a las demás variables (Cuadro 3). Este hecho podría explicarse a que el tamaño de los rebaños es relativamente grande en esos sectores, pudiendo llegar haber más de 100 crías con sus respectivas madres en un mismo corral, y se sabe que el hacinamiento y estrés de los animales predispone a la presentación de la enfermedad (De Graaf et al., 1999). En el ganado bovino se ha observado que existe una mayor frecuencia de presentación de criptosporidiosis en rebaños considerados grandes (Garber et al., 1994). Además, la crianza mixta con otras especies animales también favorece la presentación de la enfermedad (Bustinza, 2001). El sector de La Raya-IVITA, utilizado como referencia para la regresión logística múltiple, contaba con adecuado manejo higiénico sanitario, revisión semanal de las crías y rebaños de tamaño pequeño, evitando el hacinamiento y la presentación de diarrea. 


\section{Conclusiones}

- La infección por C. parvum representa un factor de riesgo para la presentación de diarrea en alpacas neonatas del departamento de Cusco.

- Las localidades de Piti, Chiaraje, La Raya-UNSAAC y Pampalacaya, presentan riesgo para la presentación de diarrea neonatal.

- Se halló un $8.6 \%$ (20/231) de animales positivos aparentemente sanos, constituyéndose en portadores asintomáticos.

\section{Agradecimientos}

Los autores agradecen a los doctores Rómulo Sapana, Dora Yucra y Danilo Pezo por su valiosa colaboración en la ejecución y revisión del presente estudio.

\section{LITERATURA CITADA}

1. Acha P, Szyfres B. 2003. Zoonosis y enfermedades transmisibles comunes al hombre y a los animales. Vol. 3. Parasitosis. $3^{\text {a }}$ ed. Lima: Organización Panamericana de la Salud. 413 p.

2. Bustinza V. 2001. La alpaca: conocimiento de gran potencial andino. Puno: Oficina de recursos del aprendizaje, Universidad Nacional del Altiplano. $495 \mathrm{p}$.

3. Caman V. 1996. Prevalencia de criptosporidiosis en alpacas neonatas en el centro alpaquero de la SAISMaranganí, Cusco. Tesis de Médico Veterinario. Lima: Facultad de Medicina Veterinaria, Univ. Nacional Mayor de San Marcos. 38 p.

4. de Graaf DC, Vanopdenbosch E, Ortega-Mora LM, Abbassi H, Peeters $J E$. 1999. A review of the importance of cryptosporidiosis in farm animals. Int J Parasitol Rev 20: 1269-1287.
5. [FAO] Food and Agriculture Organization of the United Nations. 2005. Situación actual de camélidos sudamericanos en Perú. Proyecto de cooperación técnica en apoyo a la crianza y aprovechamiento de los camélidos sudamericanos en la región andina. Lima: FAO. 63 p.

6. Fayer R, Morgan U, Upton S. 2000. Epidemiology of Cryptosporidium: transmission, detection and identification. Int J Parasitol 30: 1305-1322.

7. Foroca D, Zanabria V, Málaga J, Vilca F. 2001. Cryptosporidiosis en alpacas crías del centro de investigación y producción La Raya-UNA, Puno. Fac Med Vet Zoot, UNA 1: 3-4.

8. Garber LP, Salman MD, Hurd HS, Schlater JL. 1994. Potential risk factors for Cryptosporidium infection in dairy calves. J Am Vet Med Assoc 20: 205-210.

9. Henricksen SA, Pohlenz JFL. 1981. Staining of Cryptosporidium by a Modified Ziehl-Neelsen technique. Acta Vet Scand 22: 594-596.

10. Holland RE. 1990. Some infectious causes of diarrhea in young farm animals. Clin Microbiol Rev 10: 345-375.

11. Kuhls TL, Moiser DA, Crawford DL, Griffis J. 1994. Seroprevalencia of cryptosporidial antibodies during infancy, childhood and adolescence. Clin Infect Dis 18: 731-735.

12. López T. 1997. Estudio epidemiológico de la cryptosporidiosis en alpacas neonatas. Tesis Doctoral. León, España: Universidad de León. 173 p.

13. López T, González A, Vázquez F. 2001. Infección experimental de alpacas neonatas de alpacas con Cryptosporidium parvum. Rev Acad Per Cienc Vet 2: 11-17.

14. Lorenzo-Lorenzo M, Ares E, Villacorta I. 1993. Detection of oocyst and Ig G antibodies to Cryptopsoridium parvum in asymptomatic adult cattle. Vet Parasitol Rev 47(1-2): 9-15.

15. Ortega-Mora LM. 1996. Biología, epidemiología y control de la cryptosporidiosis. En: Resum XIII Cong Nac CiencVet. Lima. p 170-176. 
16. Ortega-Mora LM, Troncoso JM, RojoVázquez FA, Gómez-Bautista M. 1993. Serum antibody response in lambs naturally and experimentally infected with Cryptosporidium parvum. Vet Parasitol 50: 45-54.

17. Rajkhowa S, Rajkhowa C, Hazarika GC. 2006. Prevalence of Cryptosporidium parvum in mithuns (Bos frontalis) from India. Vet Parasitol 142: 146-149.

18. Rojas M. 2004. Nosoparasitosis en los rumiantes domésticos peruanos. $2^{\mathrm{a}}$ ed. Lima: Martegraf. $146 \mathrm{p}$.

19. Romero M. 1998. Prevalencia de Cryptosporidium parvum en alpacas neonatales de la sierra central peruana.
Tesis de Maestría. Lima: Facultad de Medicina Veterinaria, Univ. Nacional Mayor de San Marcos. 81 p.

20. Rothman K, Greenland S. 1998. Modern epidemiology. Boston: Lippincott-Raven. 711 p.

21. Tribeño D. 1997. Prevalencia de Cryptosporidium parvum en alpacas neonatas de Caylloma-Arequipa. Tesis de Médico Veterinario. Lima: Facultad de Medicina Veterinaria, Univ. Nacional Mayor de San Marcos. 77 p.

22. Xiao L, Fayer R, Ryan U, Upton SJ. 2004. Cryptosporidium taxonomy: recent advances and implications for public health. Clin Microbiol Rev 17(1): 72-97. 